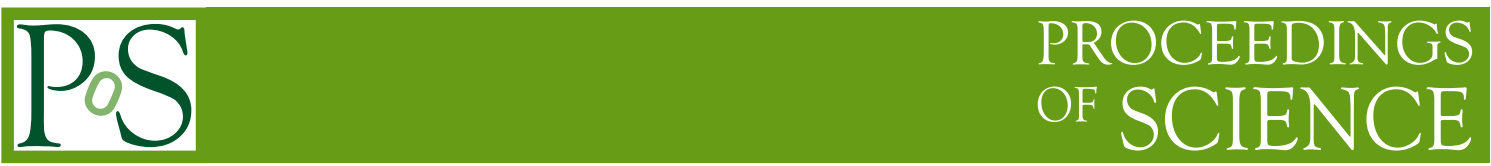

\title{
Pulsars, e-VLBI, and LEAP
}

\section{Benjamin Stappers* ${ }^{* \dagger}$}

Jodrell Bank Centre for Astrophysics, School of Physics and Astronomy, The University of

Manchester, Manchester M13 9PL. United Kingdom

E-mail: Ben. Stappers@manchester.ac.uk

\section{Michael Kramer}

Max-Planck-Institut für Radioastronomie, Auf dem Hügel 69, 53121, Bonn, Germany

E-mail: mkramer@mpifr-bonn.mpg • de

\section{Wouter Vlemmings}

Argelander Institute for Astronomy, University of Bonn, Auf dem Hügel 71, 53121 Bonn,

Germany

E-mail: woutereastro.uni-bonn.de

The compact nature of radio pulsars means that the principal aims of VLBI observations of them are to determine their distances and spatial velocities in a model independent way. There are many aspects of pulsar science which benefit from the measurement of accurate distances and velocities, but two key ones are: understanding the physics of supernovae and probing the structure of the Galaxy. Model independent velocities can be used to place strong constraints on the mechanism responsible for generating the high velocities of neutron stars. Such constraints are essential for probing the laws of physics associated with the extreme conditions that occur during supernovae. Determining distances to pulsars provides essential calibration points with which to model the Galactic electron density distribution. These models can in turn be used to improve distance estimates to pulsars without measured parallaxes. eVLBI can provide rapid observations to determine positions of newly discovered pulsars enabling efficient and rapid multi-wavelength follow-up. To enhance the sensitivity of eVLBI for pulsar observations the ability to gate the data at the pulse period is an important next step. Pulsar gating will also allow the study of any offpulse emission which might be assoicated with a pulsar wind nebula. We discuss a project allied to eVLBI, called LEAP, the Large European Array for Pulsars. This ambitious project aims to use observations of pulsars with large radio telescopes in Europe to directly detect gravitational waves. Recognising the need for a larger amount of collecting area to achieve the sensitivity required to do sufficiently high precision pulsar timing, the project will coherently combine the signals of the telescopes to form a highly sensitive tied-array beam. This project can benefit greatly from the software and technologies being developed in the e-VLBI era.

Science and Technology of Long Baseline Real-Time Interferometry:

The 8th International e-VLBI Workshop, EXPReSO9

June 22 - 262009

Madrid, Spain

\footnotetext{
* Speaker.

$\dagger$ In collaboration with the European Pulsar Timing Array Network.
} 


\section{Introduction}

Neutron stars represent one of the most extreme objects known in the Universe and they therefore provide us with an exceptional laboratory to study many aspects of physics. They are born in supernovae; explosions that mark the end of the life of a star which is around 8 times as massive as the Sun. This violent birth compresses the remaining 1.4 solar masses of material into a so-called neutron star with a radius of just $10 \mathrm{~km}$ and imparts it with a "kick" which sends itx speeding through the Galaxy with speeds greater than any other known stellar object. Many neutron stars have rotation rates of a few tens of milliseconds to seconds, very strong magnetic fields and they emit radio waves from their magnetic poles. These are the so-called pulsars, so named for the "pulse" in radio emission seen as their magnetic pole sweeps across the Earth. The large moment of inertia of these extremely dense objects mean that they have highly stable rotation rates, making them very precise clocks. Radio pulsars typically emit radio waves for just a few tens of millions of years. However, if they are in binary systems then in some cases they can accrete matter from the companion star and be spun up to millisecond periods. These "recycled pulsars" are reactivated and once again emit radio waves. They are by far the most stable rotators of all the pulsars and are ideal for performing tests of theories of gravity and detecting gravitational waves.

Knowing the positions, space velocities and distances to pulsars are vital at all stages of their evolution. In the case of young pulsars we can use accurate positions and velocities to associate them with the supernova remnants which mark their birth location [1], or if they are somewhat older and the supernova remnant has faded we can associate them with the birth sites of neutron stars, like OB associations [2]. This provides vital input for understanding the physics of the supernova explosion in which they formed [3] and the evolution of massive stars. Getting accurate distances allows us to know intrinsic properties of the pulsars such as their radio luminosities and in the case of thermal X-ray emitters, their temperature and thus information on the cooling of neutron stars.

As they traverse the distance from the pulsar to the Earth the free electrons in the interstellar medium impart a frequency dependent dispersive delay which causes lower frequencies to arrive later than high frequencies, smearing out the pulse profile. Moreover the combined effect of the electrons and the Galactic magnetic field imparts a frequency dependent rotation on the plane of polarisation of the radio emission. Having sufficiently large numbers of pulsars with accurate distances one can build up models of the free electron [4] and the magnetic field distribution (e.g. [5]) in the Galaxy. The former can then be used to better determine distances to pulsars where only the dispersive delay is known, while both can be used to improve our understanding of the formation and evolution of the Galaxy.

We present just a sample of the possible applications of accurate pulsar distances and velocities. However one other is of specific importance. Pulsars in binary systems with other neutron stars are extremely good for tests of gravity. In some cases though the precision of those tests is limited by the unknown distance, as is the case for PSR B1534+12 [6] and also in the best system for these tests, the double pulsar [7].

The stable rotation rates of pulsars means that in some cases we can measure the proper motion and parallax for radio pulsars using pulsar timing. However young pulsars often aren't stable enough rotatos, due to glitches or timing noise, to enable the measurement of proper motion and certainly not parallax. The timing signature of parallax depends on measuring the curvature of the 
wavefronts as they arrive at the Earth's orbit and is a very weak effect. It has therefore only been measured for a handful of millisecond pulsars. Thus eVLBI has a very important role to play in determining the distances and velocities of greater samples of both young and millisecond pulsars.

Gravitational waves are a fundamental part of Einstein's theory of General Relativity (GR) but they have, to date, never been directly detected. However, there is clear indirect evidence that they exist, as shown by the observed orbital period decay of the double neutron star binary PSR B1913+16 matching exactly the predictions of GR [8] and even more exactly with the double pulsar PSR J0737-3039A/B [9]. There are a number of proposed and running experiments which aim to detect gravitational waves directly, for example LIGO and LISA, both of which principally aim to detect short orbital period, or inspiralling, binaries. Radio pulsars can also be used to detect gravitational waves in a frequency range of a few nanohertz, where no other yet conceived experiment could detect them. This frequency range is of particular interest because it typically relates to events early in the history of the universe such as merging supermassive black holes and cosmic strings. Using radio pulsars to try and detect gravitational waves is not a new idea but there is growing evidence that it can be done (e.g. [10]). One of the major limitations presently encountered simply seems to be that of sensitivity and so lots of observing time on very large telescopes is required.

\section{2. eVLBI}

One of the principal ways in which eVLBI can be extremely useful is in accurately determining positions of newly discovered pulsars. As discussed above, using pulsar timing means that up to a year is required to get an accurate position and if there is significant timing noise it may never be possible. As more and more pulsars are found, and in particular in crowded regions of the sky like the Galactic plane, the accuracy of positions required increases. Accurate positions for young, energetic pulsars are vital in order to allow multi-wavelength follow-up, in particular at high energies and especially if the high energy emission is not pulsed. In the case of millisecond pulsars an early determination of the position allows more rapid searches for any binary companions to be made, thus more rapidly illuminating the nature of the binary. The likely further increase in the number of pulsars known also makes it difficult to get sufficient time to determine period derivatives, and hence their nature, on a reasonable timescale. An accurate position from eVLBI can lead to a rapid determination of whether the pulsar is particularly interesting, i.e young and energetic or high magnetic field, and thus whether it is worthy of further detailed study.

There are presently many surveys for new pulsars being carried out or about to start. These surveys span both hemispheres and will include all-sky surveys. The new pulsars they find, combined with those already found in the Parkes Multibeam surveys, provide us with many more lines of sight through the interstellar medium. There is therefore a great opportunity to use these new pulsars as probes of the interstellar medium by measuring their parallaxes. In particular the LOFAR all sky survey is likely to find many weak nearby pulsars and many at higher Galactic latitudes, providing sources to constrain the nearby and halo electron content and magnetic field structure.

An area where the flexibility of data analysis that might be afforded by future developments in eVLBI is the use of pulsar gating. In pulsar gating the correlated data are divided up in time bins which span the pulsar period based on the known ephemeris. The data are then averaged and one 
can make images of a particular pulse longitude. Most usually this is done to make an "on" and an "off" image. This has been successfully done in VLBI with the VLBA for example but not yet with European correlators. The advantage of gating for most applications is that it allows one to improve the signal-to-noise of the detection by the square root of the duty cycle of the pulsar. It also provides an excellent way to study any (relatively) compact underlying emission that might be associated with a pulsar wind nebula. Gated observations may also provide evidence for so-called "off pulse" radio emission that might be associated with the sites of high energy emission such as that seen by the FERMI gamma-ray telescope. Detecting pulsar wind nebulae in the radio are interesting because they can be combined with detections at other wavelengths to perform calorimetry. The shocks are expected to be small in the radio, but it is possible with eVLBI to resolve the inner shocks. Radio observations also probe different ages and energetics of electrons.

\section{Current status and Near Future}

Over the last few years there has been excellent progress in getting new and improved values for both proper motions and parallaxes. A VLBA based project has measured 14 new proper motions and parallaxes which includes the highest directly detected velocity (PSR B1508+55; [3]) and the furthest model independent distance (PSR B1541+09; [11]). An LBA project in the southern hemisphere has measured 7 new parallaxes and proper motions [12] and in particular of the double pulsar (see above). In the near future we will be undertaking an eMerlin Legacy project, called $\mathrm{e} \pi$, to observe a sample of up to 60 pulsars. This will include some observations at C-band and others at L-band and will use the greatly enhanced bandwidth of eMerlin combined with pulsar gating to allow us to probe weaker nearby pulsars and brighter more distant ones.

\section{LEAP}

The LEAP project is based around the fact that in order to detect gravitational waves with radio pulsars we need to improve the timing precision that we can achieve. Recent work has shown that one can do extremely well with individual $100 \mathrm{~m}$ class telescopes with state-of-the art hardware. However it seems that there just aren't enough bright pulsars and insufficient numbers of them are visible from Arecibo. We recognised a few years ago that there are a number of $100 \mathrm{~m}$ dishes in Europe which are performing precision pulsar timing experiments and we formed the European Pulsar Timing Array (EPTA). The EPTA can potentially do better than other pulsar timing array projects in that it has more measurements overall, can more easily do multi-frequency observations over a wide range of frequencies, and has a natural way to check on clock and instrumental based errors. It already shares the timing data from the individual telescopes and this has led to an improvement in our ability to study individual systems and is presently being used to place a first European telescope based limit on the gravitational wave background.

However, it is possible to do even better than this by using VLBI-like techniques. The idea is to combine the signals from all the telescopes coherently by using phase solutions based on observations of nearby calibrator sources. Thus, by combining data from the Lovell Telescope in the United Kingdom, the WSRT in the Netherlands, the Effelsberg Telescope in Germany, the Nançay Telescope in France and the soon to be completed Sardinia Telescope in Italy we can 


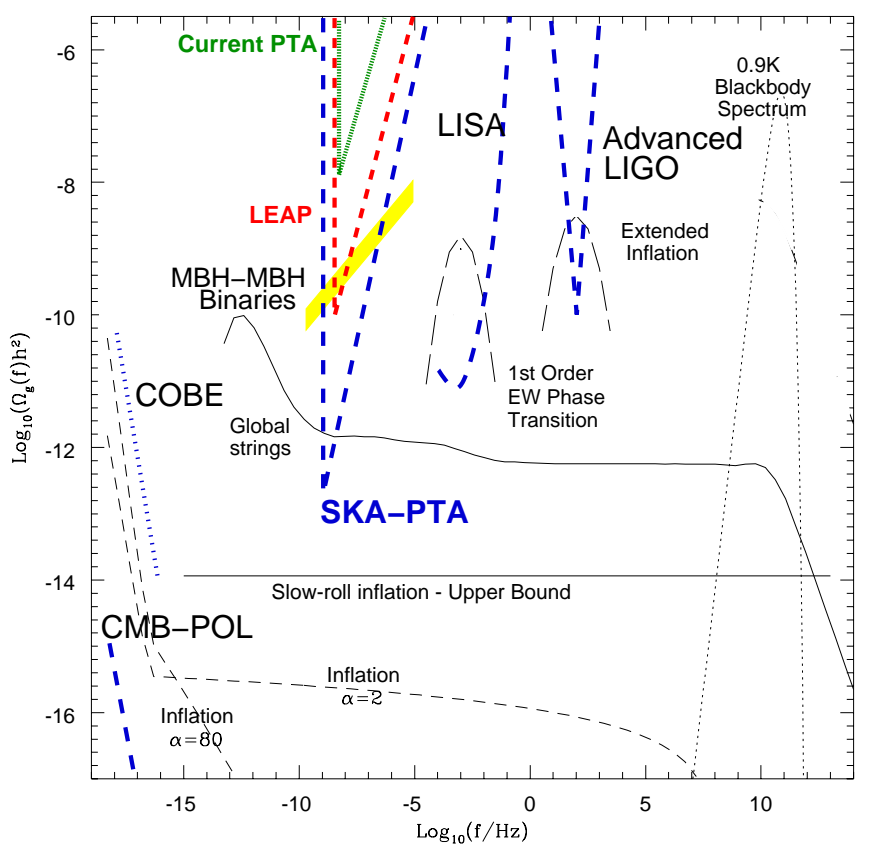

Figure 1: Summary of the potential cosmological sources of a stochastic gravitational wave (GW) background overlaid with bounds from COBE, current Pulsar Timing Array (PTA) experiments and CMB polarization goals, LISA and Advanced LIGO. LEAP will improve on the current best PTA limits by more than two orders of magnitude, enabling the detection of a GW background caused by the merger of massive black holes (MBHs) in early galaxy formation.

synthesize a telescope of approximately $194 \mathrm{~m}$ in diameter. This corresponds to by far the largest steerable high frequency radio telescope in the world. This project is funded through a European Research Council Advanced Grant to Michael Kramer.

The present plan is that at least $100 \mathrm{MHz}$ of bandwidth at L-band will be baseband recorded to disks at each of the five telescopes. Calibration sessions will be carried out before and after each observation so as to determine the phases in order to be able to combine the data. The observing sessions will be up to 24 hours in duration, generating about 34.5 Terabytes of data from each telescope. The disks with the data will be transferred to Jodrell Bank where a cluster of computers will be used to produce the coherent time series, this will then be fed to "standard" radio pulsar processing software to generate times of arrival from each pulsar. These very high precision data will be supplemented with multi-frequency data from the continuation of the existing pulsar timing programs on each of the individual telescopes.

eVLBI can be highly beneficial to this project and the future of high precision timing. In the initial stages the significant expertise in the community will be a great resource to help with the issues that will be encountered. We are already investigating the software correlators devloped for eVLBI, e.g DiFX [13] and one by JIVE (sfxc). In particular with the use of non-heterogeneous observing systems like we will have. A particular exciting possibility would be to eventually move away from using disk based systems and taking advantage of the network connections already developed for eVLBI. The data rates that we are considering for LEAP are presently somewhat higher than what is possible, but not too far that it might not be possible in a few years. The other interesting possibility is to develop the ability to take the development work that will be undertaken 
in LEAP and make it part of a future eVLBI correlator. This would mean that resources can be used most effectively and also allow the possibility of expanding LEAP beyond the current 5 telescopes.

\section{Conclusions}

A wish list of how eVLBI already can and might in the future enhance pulsar observations would include; a rapid turn around between observations and data acquisition, excellent sensitivity, pulsar binning mode to win 2-5 times more sensitivity, sufficiently large numbers of frequency channels to allow for dedispersion to enhance the gating, regular sessions to allow for rapid follow up of new discoveries and the increased sensitivity to have more in beam calibrators.

The LEAP project has the potential to revolutionise high precision pulsar timing and it will use VLBI techniques to achieve that goal. Incorporating the pulsar timing mode into future eVLBI correlating capabilities may allow this practice to become even more routine and allow for enhancements by including even more telescopes.

\section{References}

[1] Winkler, P. F. and Petre, R. Direct Measurement of Neutron Star Recoil in the Oxygen-rich Supernova Remnant Puppis A, ApJ 670 (2007)

[2] Vlemmings, W. H. T., Cordes, J. M., and Chatterjee, S. Separated at Birth: The Origin of the Pulsars B2020+28 and B2021+51 in the Cygnus Superbubble, ApJ 610 (2004)

[3] Chatterjee, S., Vlemmings, W. H. T., Brisken, W. F., Lazio, T. J. W., Cordes, J. M., Goss, W. M., Thorsett, S. E., Fomalont, E. B., Lyne, A. G., and Kramer, M., Getting Its Kicks: A VLBA Parallax for the Hyperfast Pulsar B1508+55, ApJ Lett 630 (2005)

[4] Cordes, J. M. and Lazio, T. J. W., NE2001.I. A New Model for the Galactic Distribution of Free Electrons and its Fluctuations, ArXiv e-print, astro-ph/0207156 (2002)

[5] Noutsos, A., Johnston, S., Kramer, M.and Karastergiou, A., New pulsar rotation measures and the Galactic magnetic field, MNRAS 386 (2008)

[6] Stairs, I.H., Thorsett, S.E, Taylor, J.H. and Wolszczan, A., Studies of the Relativistic Binary Pulsar PSR B1534+12. I. Timing Analysis, ApJ 581 (2002)

[7] Deller, A. T.; Bailes, M.; and Tingay, S. J., Implications of a VLBI Distance to the Double Pulsar J0737-3039A/B, Science 323 (2009)

[8] Taylor, J. H., Fowler, L. A. and McCulloch, P. M., Measurements of general relativistic effects in the binary pulsar PSR 1913+16 Nature 277 (1979)

[9] Kramer, M. et al, Tests of General Relativity from Timing the Double Pulsar, Science 314 (2006)

[10] Jenet, F. A., et al, Upper Bounds on the Low-Frequency Stochastic Gravitational Wave Background from Pulsar Timing Observations: Current Limits and Future Prospects, ApJ 653 (2006)

[11] Chatterjee, S., et al, Precision Astrometry with the Very Long Baseline Array: Parallaxes and Proper Motions for 14 Pulsars, ApJ 698 (2009)

[12] Deller, A. T.; Tingay, S. J.; Bailes, M. and Reynolds, J. E., Precision Southern Hemisphere VLBI Pulsar Astrometry. II. Measurement of Seven Parallaxes, ApJ 701 (2009)

[13] Deller, A. T.; Tingay, S. J.; Bailes, M.; West, C., DiFX: A Software Correlator for Very Long Baseline Interferometry Using Multiprocessor Computing Environments, PASP 119 (2007) 